\title{
Volunteers trained in CPR and use of automated external defibrillators increased survival after out of hospital cardiac arrest
}

Hallstrom AP, Ornato JP, Weisfeldt M, et al. Public-access defibrillation and survival after out-of-hospital cardiac arrest. N Engl J Med 2004;351:637-46.

\section{Q Do more patients with out of hospital cardiac arrest survive to hospital discharge when response teams of lay volunteers trained in cardiopulmonary resuscitation (CPR) also use automated external defibrillators (AEDs)?}

\section{METHODS}

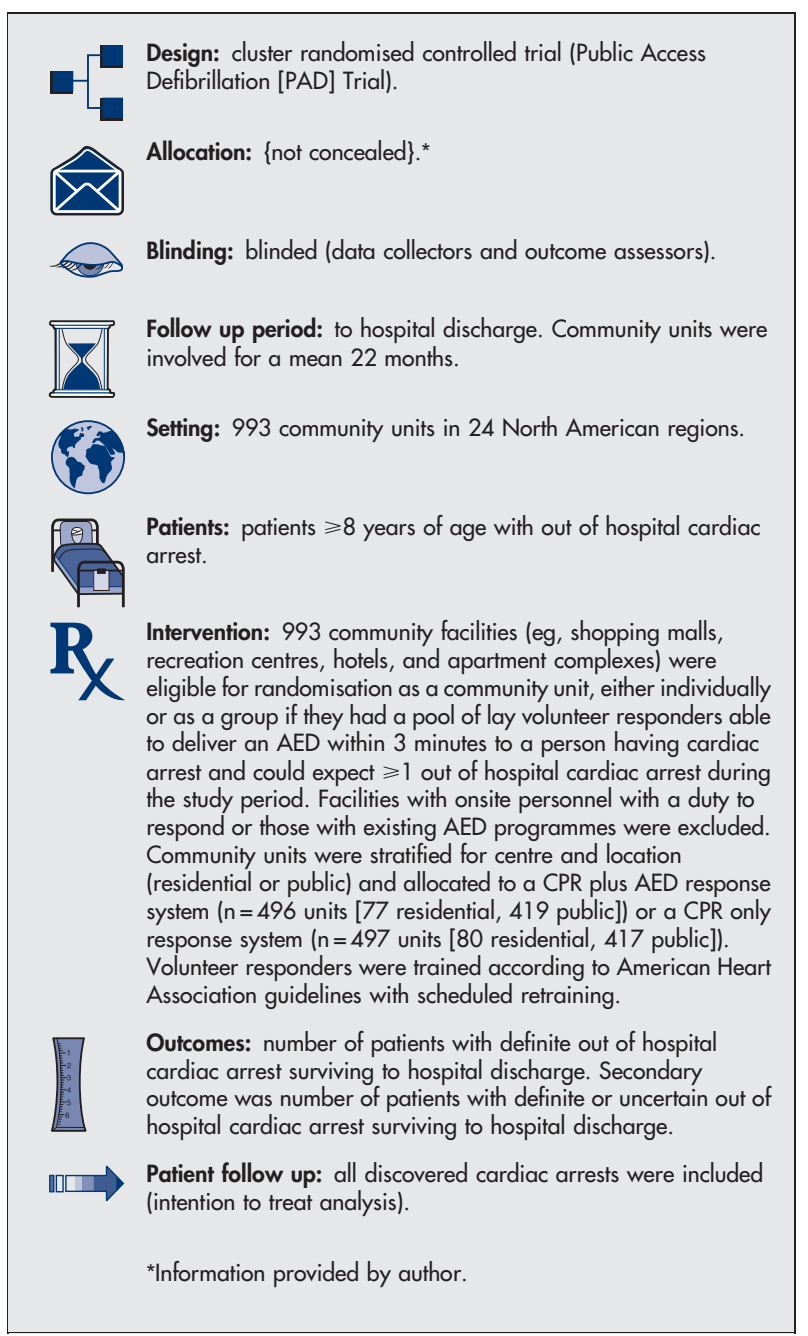

For correspondence: Dr A Hallstrom, Public Access Defibrillation Clinical Trial Center, University of Washington, Seattle, WA, USA. padctc@ u.washington.edu

Sources of funding: National Heart, Lung and Blood Institute; American Heart Association; Guidant Foundation; Medtronic. Devices supplied by Cardiac Science Survivalink; Medtronic Physio-Control; Philips Medical Systems Heartstream; Laerdal Medical.

\section{MAIN RESULTS}

235 cardiac arrests were classified as definite, and 4 were classified as probable or uncertain. The number of definite out of hospital cardiac arrests was 128 in the CPR plus AED group and 107 in the CPR only group $(p=0.09)$. More patients in the CPR plus AED group survived to hospital discharge than patients in the CPR only group (30 v 15, relative risk 2.0, 95\% CI 1.07 to 3.77). All but 2 survivors ( 1 in each group) were in public units.

\section{CONCLUSION}

Among patients with out of hospital cardiac arrest, more survived to hospital discharge when response teams of lay volunteers trained in cardiopulmonary resuscitation also used automated external defibrillators.

A modified version of this abstract appears in ACP Journal Club.

\section{Commentary}

revious studies have shown that use of AEDs significantly improves outcomes for in hospital and out of hospital cardiac arrest. ${ }^{12}$

The PAD Trial investigators studied the difference in outcome delivered by adding use of AEDs to training lay personnel in diverse community settings. Volunteers within these settings were trained according to American Heart Association guidelines for CPR and emergency cardiovascular care. The findings show that teaching CPR plus AED use will increase survival after cardiac arrest.

As is relatively common in clinical trials, the study observed much higher than anticipated rates of survival in the standard care (CPR only) group. This suggests that the trial has underestimated the effect likely to be found when implementing AED use in real world settings.

AEDs seem to be safe in the hands of lay volunteers; no inappropriate shocks were given, and any adverse events were related to transient psychological trauma to volunteers and stolen AEDs. It is also noteworthy that the groups did not differ for cerebral performance at discharge. However, the study was not powered for this outcome, so the finding should be viewed cautiously.

The PAD trial does not address the many cardiac arrests that occur in the home, and the findings should not be considered relevant to this population. However, the findings are relevant to facilities likely to service large volumes of people $>35$ years of age: retirement villages, cardiac rehabilitation programmes, large factories, major transport nodes (eg, airports and central railway stations), shopping centres, and swimming pools are some of the settings that should consider the purchase of AEDs in addition to maintaining trained response teams that receive regular updates. Where facilities already have response teams, the marginal cost of purchasing AEDs will be relatively low when all costs are considered. Sidney Cuthbertson, RGON, MHSc, CCNS Auckland District Health Board Auckland, New Zealand

1 Atkins DL, Hartley LL, York DK. Accurate recognition and effective treatment of ventricular fibrillation by automated external defibrillators in adolescents. Pediatrics 1998; 101:393-7.

2 Eisenberg MS. Is it time for over-the-counter defibrillators? JAMA 2000;284:1435-8. 